\title{
Accelerators
}

\section{The Future Circular Collider study}

\section{An international study has been launched to examine options for a circular collider at CERN in the post-LHC era.}

More than 350 world experts in accelerators and particle physics, including several laboratory directors, came together at the University of Geneva on 12-15 February to launch the Future Circular Collider (FCC) study, which will examine options for an energy-frontier collider based on a new 80-100-km-circumference tunnel infrastructure. The FCC study, which will be organized as a worldwide international collaboration, comprises a $100 \mathrm{TeV}$ proton (and heavy-ion) collider at the energy frontier, a high-luminosity $\mathrm{e}^{+} \mathrm{e}^{-}(\mathrm{H}, \mathrm{Z}, \mathrm{W}$, and $\mathrm{t} \overline{\mathrm{t}})$ factory as a potential intermediate step, and an analysis of options for a hadron-lepton collider. The goal of the study is to deliver a conceptual design report (CDR) together with a cost review by 2018, in time for the next update of the European Strategy for Particle Physics. The CDR will integrate physics, detector, accelerator and infrastructure aspects.

The FCC design study responds to a high-priority request in the 2013 update of the European Strategy for Particle Physics (CERN Courier July/August 2013 p9) stating that "A conceptual design study of options for a future high-energy frontier circular collider at CERN for the post-LHC era shall be carried out". February's kick-off meeting was co-sponsored by the Extreme Beams work package 5 of the EuCARD-2 project, within the European Commission's FP7 Capacities Programme. Participants came from all over the world, with particularly strong representation from China, Japan, Russia and the US, in addition to the many attendees from laboratories and universities across Europe. The goals of the meeting were to introduce the FCC study, to discuss its scope and organization, and to prepare and establish global collaborations.

In his opening address, CERN's director-general, Rolf Heuer, presented an exciting perspective and explained the main motivations for the FCC, while also cautioning that it was too early to make any cost estimate. Nima ArkaniHamed of the Institute for Advanced Study in

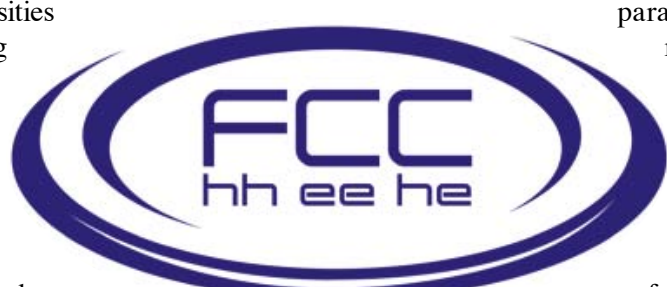

Princeton, and recently appointed as the first director of the Centre for Future High Energy Physics at the Institute of High Energy Physics (IHEP) in Beijing, highlighted the compelling physics case for the $100 \mathrm{TeV}$ hadron collider. Precision physics will be essential at both the lepton and hadron colliders, as Christoph Grojean from the Institut de Física d'Altes Energies in Barcelona underlined.

A similar study for a $50-70 \mathrm{~km}$, double-purpose lepton and hadron collider is being pursued in China, with an attractive site proposal and ambitious schedule. In presenting the project, Yifang Wang, director of IHEP in Beijing, conceded that it would be a difficult project but it would also be very exciting. Even if implemented somewhere other than in China, it would still be beneficial to the field of particle physics in general and to the Chinese high-energy physics and scientific community in particular. To this end, IHEP fully supports a global effort. Fermilab's associate director for accelerators, Stuart Henderson, also reported a broad acknowledgement in the US that any future collider would need to be a global enterprise, requiring financial and human resources from across the world. He stressed that the US community wishes to play a role in any future collider, while also mentioning several domestic "grass-roots" activities.

Frédérick Bordry, CERN's director of accelerators and technology, presented the roadmap for CERN. Europe's top priority for the next two decades is the exploitation of the LHC, with nominal parameters and a total integrated luminosity of about $300 \mathrm{fb}^{-1}$ by 2023 , and with the High-Luminosity LHC upgrade to reach $3000 \mathrm{fb}^{-1}$ by 2035 (CERN Courier January/February 2014 p12 and p23). In parallel, as one of the next-highest-priority items, the FCC design study will be pursued along with CLIC as a potential post-LHC accelerator project at CERN. Michael Benedikt, the

FCC study co-ordinator, reviewed the baseline parameters, design challenges and preparations for global collaboration, stressing that new partner institutes will be welcome throughout the duration of the study. Key technologies are high-field magnets for the hadron collider and an efficient high-power superconducting RF (SRF) system for the lepton collider. Possible R\&D The FCC study logo reflects the three different colliders that could be housed in the new tunnel. goals for the study include the development of short 16-T dipole models in all regions (America, Asia and Europe) by 2018 and, in parallel, demonstration of 20-T magnet 


\section{Accelerators}

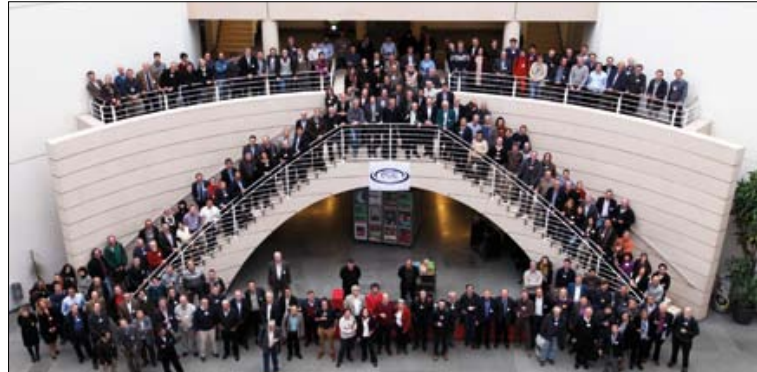

Participants of the kick-off meeting at the University of Geneva. (Image credit: Michael Hoch/CERN.)

technology based on the combination of high- and low-temperature superconductors as well as SRF developments, targeted at overall optimization of system efficiency and cost.

Philippe Lebrun, former head of CERN's Accelerator Technology Department, pointed out that, although CERN's experience in building machines of increasing size and performance can be applied to the study of $80-100 \mathrm{~km}$ circular accelerators in the Geneva basin, the step from the $27 \mathrm{~km}$ Large Electron-Positron collider and the LHC to the FCC represents major challenges. These will require inventive solutions in accelerator science and technology as well as in conventional facilities. Felix Amberg from Amberg Engineering - a company involved in the Gotthard Base Tunnel project-reported and analysed specific aspects of building long tunnels. His presentation suggested that tunnelling costs and risks can be predicted fairly reliably, provided that the project does not extend over too long a time interval and that the legal framework remains stable during the construction period.

After two days of plenary sessions, which surveyed the scope, plan, international situation and design starting points of the FCC, seven parallel sessions gave space for feedback, additional presentations and lively international discussions. Worldwide collaboration in all areas - physics, experiments and accelerators - was found to be essential to reach the level for a CDR by 2018. Key R\&D areas for the FCC, such as superconducting high-field magnets and SRF, are of general interest and relevant for many other applications. Significant R\&D investments have been made over the past decade(s), for example in the framework of the LHC and High-Luminosity LHC. Further continuation will ensure efficient use of these investments. At the kick-off meeting a consensus emerged on the approach to form a global collaboration for this study, and many participants expressed a strong interest - both for themselves and their institutes.

Institutes worldwide are now invited to join the global FCC effort, and to submit non-committing written "expressions of interest" with regard to specific contributions by the end of May 2014 .

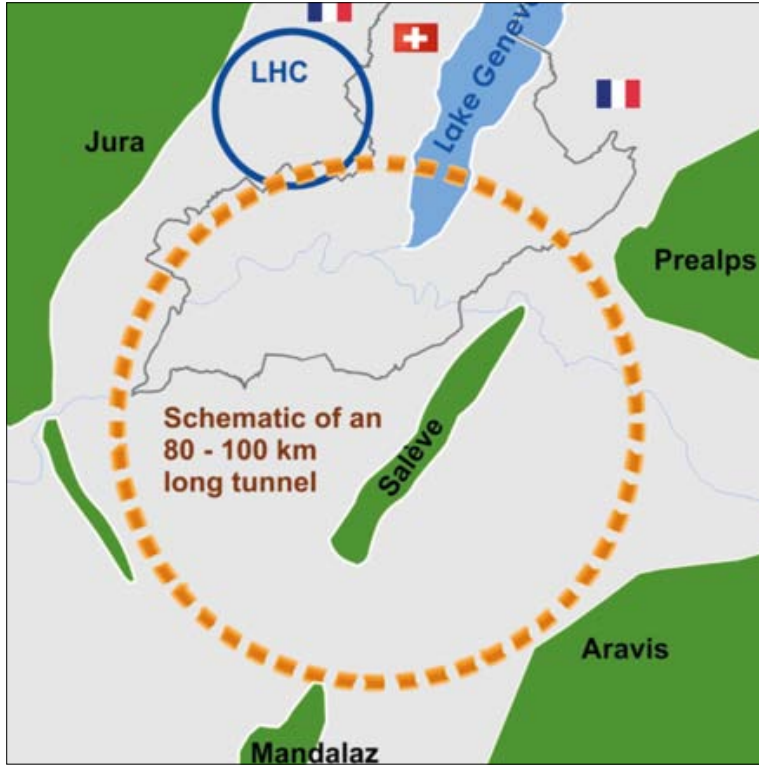

Sketch of a future 80-100-km-long tunnel in the Geneva area, which would allow for a 100-TeV, energy-frontier proton collider and also, as a potential intermediate step, a high-luminosity $e^{+} e^{-}$collider serving as a W, Z, H and $t \bar{t}$ factory. (Image credit: CERN.)

\section{- Further reading}

For all of the presentations, see http://indico.cern.ch/e/fcc-kickoff/.

\section{Résumé}

Étude sur le futur collisionneur circulaire

Une étude internationale a été lancée afin d'examiner les options possibles concernant un futur collisionneur circulaire de haute énergie au CERN, qui serait abrité dans un tunnel de 80 à $100 \mathrm{~km}$ de circonférence. Cette étude, qui sera menée dans le cadre d'une collaboration internationale planétaire, portera sur un collisionneur hadronique de haute énergie de $100 \mathrm{TeV}$, un collisionneur $e^{+} e^{-}$haute luminosité qui servirait d'étape intermédiaire et permettrait d'étudier les options possibles pour un collisionneur hadron-lepton. L'objectif est d'élaborer un rapport préliminaire de conception (CDR) et une analyse des coûts d'ici à 2018, à temps pour la prochaine mise à jour de la stratégie européenne pour la physique des particules.

\section{Michael Benedikt and Frank Zimmermann, CERN.}


EDOdFElloW

www.goodfellow.com

Metals

and materials for research

Goodfellow Cambridge Limited

Ermine Business Park

Huntingdon PE29 6WR UK

Tel: 08007314653 or +441480424800

Fax: 08003287689 or +441480424900

info@goodfellow.com

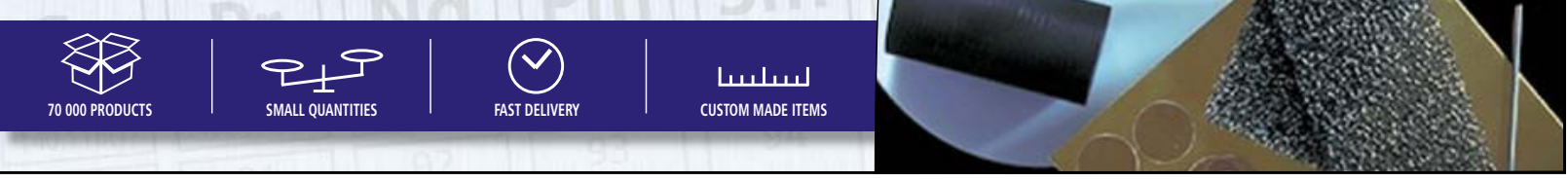

\section{VERIFY AND OPTIMIZE YOUR DESIGNS WITH COMSOL MULTIPHYSICS}

RF COUPLER: This model computes the transmission probability through an RF coupler using both the angular coefficient method available in the Free Molecular Flow interface and a Monte Carlo method using the Mathematical Particle Tracing interface.

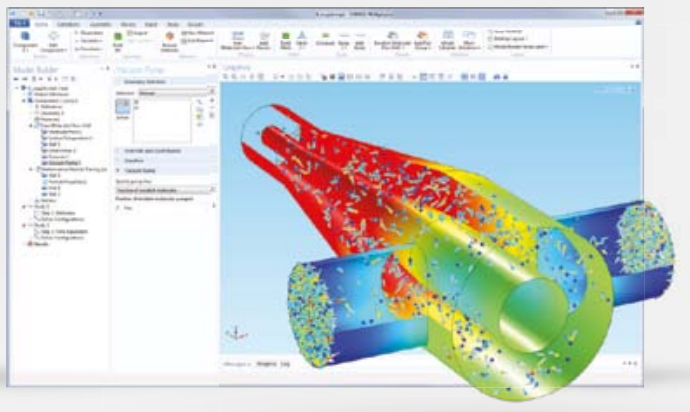

Multiphysics tools let you build simulations that accurately replicate the important characteristics of your designs. The key is the ability to include all physical effects that exist in the real world.

To learn more about COMSOL Multiphysics, visit www.comsol.com/introvideo 